\title{
Ages and Metallicities of Globular Clusters in NGC 5128
}

\author{
Enrico V. Held \\ Osservatorio Astronomico di Padova, vicolo dell'Osservatorio 5, I-35122 \\ Padova, Italy \\ Luciana Federici, Carla Cacciari \\ Osservatorio Astronomico di Bologna, via Ranzani 1, I-40127 Bologna, \\ Italy \\ Vincenzo Testa \\ Osservatorio Astronomico di Roma, via Frascati 33, I-00040 Monte \\ Porzio Catone, Italy
}

\begin{abstract}
We have obtained multislit spectroscopy of 40 known globular clusters in the nearby peculiar elliptical galaxy NGC 5128 at the ESO NTT telescope. Measurements of metal and hydrogen absorption line indices have been used to estimate the age and metal content of individual clusters. The results have been combined with previous data from multifibre spectroscopy of clusters in the halo of NGC 5128 to study the age and metallicity distribution of the globular cluster system.
\end{abstract}

\section{Introduction}

Spectroscopy of extragalactic globular clusters provides a wealth of information on the evolution of their parent galaxies. Studies of the globular cluster systems may help us to understand the processes of galaxy formation, since there is increasing evidence that clusters formed both at early times and during secondary cluster formation episodes, possibly (but not necessarily) related to galaxy mergers and interactions.

NGC 5128 provides the nearest example of a globular cluster system in a giant elliptical galaxy. Its inner regions harbor a disk of dust, gas and young stars, possibly the consequence of a merger with a late-type spiral galaxy which occurred some $10^{8}-10^{9}$ yr ago (e.g., Israel 1998). The globular cluster population of NGC 5128 has been the subject of several studies. Harris et al. (1992) presented a study of 62 spectroscopically confirmed clusters using photometry on the Washington system. That work indicated a large range in metallicity with a mean abundance of $[\mathrm{Fe} / \mathrm{H}]=-0.8 \pm 0.2 \mathrm{dex}$, and a weak spatial metallicity gradient. By analyzing the same data, Zepf \& Ashman (1993) suggested the color histogram to be best fitted by a bimodal metallicity distribution, indicative of a secondary cluster generation thought to be a natural consequence of galaxy mergers. Recently, Rejkuba (2001; also this conference) obtained optical and near-IR photometry of a new deep sample of candidate clusters in two fields 
at $9^{\prime}$ and $14^{\prime}$ from the galaxy center. Her results based on $(U-V)$ colors also support a bimodal metallicity distribution. The presence of a detectable metallicity gradient among the inner clusters was also indicated by HST/NICMOS observations of candidate clusters in the inner $3 \mathrm{Kpc}$ of NGC 5128 (Minniti et al. 1996).

The main source of uncertainty in the photometric data are the poorly known effects of internal reddening, a problem overcome by spectroscopy using absorption line indices. Jablonka et al. (1996) addressed the problem of whether super-metal-rich clusters exist in NGC 5128, as the infrared photometry of Frogel (1984) might suggest. Spectrophotometry of the five reddest clusters led to the conclusions that no cluster has metallicity higher than solar.

Recent HST color-magnitude diagrams of halo fields in NGC 5128 (Soria et al. 1996; Harris \& Harris 2000; see also W. Harris, this conference) provide the possibility to compare the metallicity distributions of globular clusters and halo stars in NGC 5128. Understanding the relationship between the formation history of halo stars and globular clusters may be a clue to our understanding of the evolution of elliptical galaxies.

\section{Observations and data analysis}

Multi-object spectroscopy of 40 globular clusters in NGC 5128 was obtained on the nights of 2-3 April 1998 with the imaging spectrograph EMMI at the ESO NTT telescope. Two MOS masks were observed each night, with typical exposure times of 3 hours each. The masks were designed to include the Lick indices $\mathrm{H} \beta, \mathrm{Fe} 50, \mathrm{Mg} 1, \mathrm{Mg} 2, \mathrm{Mg} b, \mathrm{Fe} 52$, and $\mathrm{Fe} 53$ for all objects. $\mathrm{H} \gamma$ is also present in most spectra. The MOS sample was selected from the list of Harris et al. (1992), including clusters which have membership confirmed by previous spectroscopic work of Hesser et al. (1986) and Sharples (1988). Long-slit spectra of Galactic globular clusters (GGC) in a wide range of $[\mathrm{Fe} / \mathrm{H}]$ were also observed as metallicity templates. The strengths of Balmer and metal absorption lines were measured using the index definitions of Worthey et al. (1994), and the errors were accurately estimated by error propagation throughout the data reduction. Well defined correlations were found between metal line indices for the NGC 5128 clusters. The relative strengths of $\mathrm{Fe}$ and $\mathrm{Mg}$ features follow the same relations as for Galactic globular clusters.

\section{Ages and metallicities}

Plots of Balmer line intensities against metal indices were used to estimate ages and metallicities for the NGC 5128 clusters. Figure 1 shows our NTT data plotted in the $\mathrm{H} \beta$ vs. $\mathrm{Mg} 2$ diagram, along with a grid of spectrophotometric models from Bressan et al. (1996). In general, the globular clusters in NGC 5128 appear consistent with old Galactic globulars. The scatter in the diagram is that expected from measurement errors. For two clusters, however, both $\mathrm{H} \gamma$ and $\mathrm{H} \beta$ are much stronger than predicted for an old, metal-poor population. Comparison with the models suggests that both clusters are younger than 1 Gyr. It is intriguing to note that this estimated age is close to the epoch when a spiral companion might have been captured and merged with NGC 5128 (e.g., 


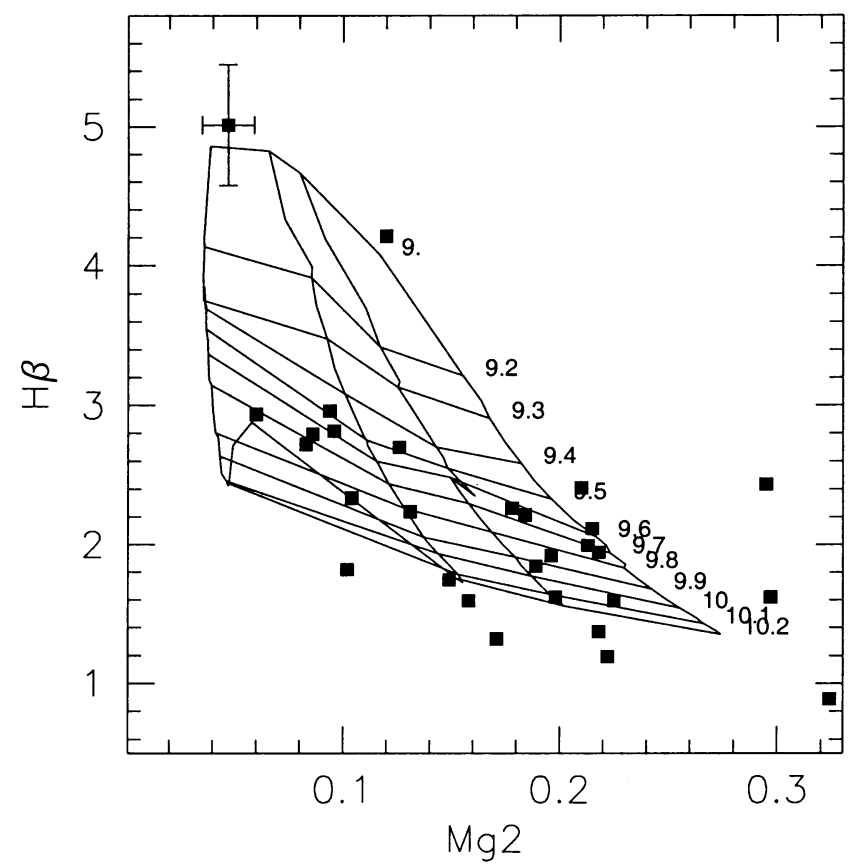

Figure 1. $\quad \mathrm{H} \beta$ versus Mg2 line strengths for the NTT sample of globular clusters in NGC 5128, with a superimposed grid of model line strengths from the simple stellar population models of Bressan et al. (1996), with $Z=0.0004,0.004,0.008$, and 0.02 (left to right). The nearly horizontal lines indicate models of equal age from 1 to $16 \mathrm{Gyr}$ (the labels are logarithm of age). The error bar is representative of the typical errors on the spectrophotometric indices.

Israel 1998). A young age for these two clusters is also confirmed by their blue colors, pointing to the presence of a blue continuum from young stars (cf. Zepf $\&$ Ashman 1993). For one of the two, the integrated $\left(C-T_{1}\right)$ color implied an unrealistic low metallicity $[\mathrm{Fe} / \mathrm{H}]=-3.3$.

For individual clusters, mean $[\mathrm{Fe} / \mathrm{H}]$ values were obtained by robust estimation of the average from several metal line indices. Empirical index-metallicity relations were obtained by plotting metal line indices for Galactic globular clusters against GGC metallicities on the Zinn \& West (1984) scale. For the old clusters in NGC 5128, these empirical calibrations provide the most reliable estimates of metal abundance. For young clusters, however, the metallicities obtained by empirical calibration are underestimated, and proper interpolation on the model grids is required to derive both ages and metallicities. 


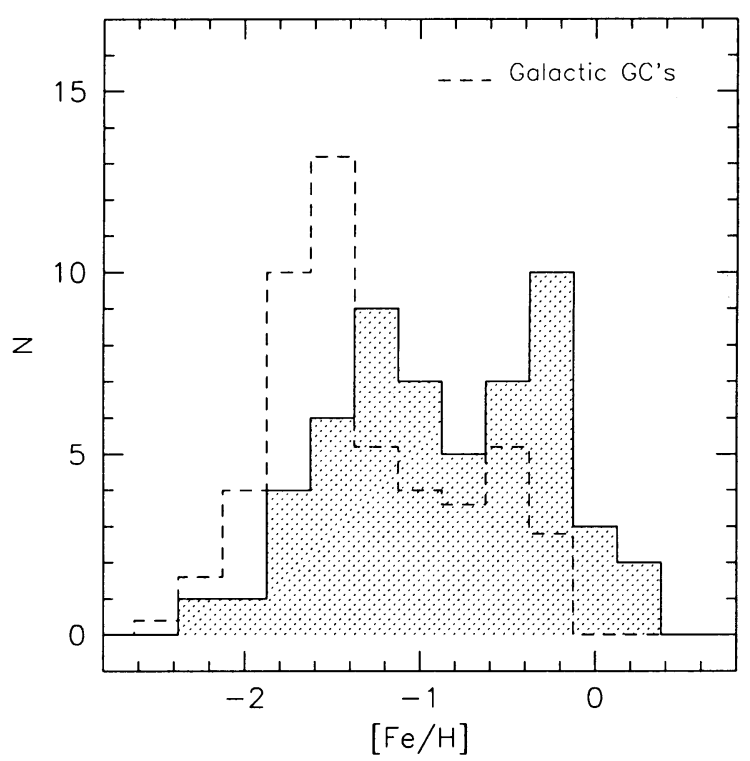

Figure 2. The metallicity distribution of NGC 5128 globular clusters in our spectroscopic sample, compared with the abundance distribution of Galactic globular clusters.

Figure 2 presents the distribution of the spectroscopically derived metallicities for 55 clusters in NGC 5128 for which we have so far obtained reliable absorption line measurements, including the results of previous multifibre spectroscopy (Held et al. 1997). The globular clusters in NGC 5128 span a range in metallicity from $[\mathrm{Fe} / \mathrm{H}] \sim-2$ to slightly above solar, with a mean value $<[\mathrm{Fe} / \mathrm{H}]>\approx-0.9$ and clear evidence for a bimodal distribution. The peaks at $[\mathrm{Fe} / \mathrm{H}] \sim-1.2$ and $[\mathrm{Fe} / \mathrm{H}] \sim-0.3$ are in good agreement with the results from photometry.

Overall, the metallicity of the globular cluster system of NGC 5128 is higher than in the Milky Way, and comparable to that inferred for other giant ellipticals (e.g., Kissler-Patig et al. 1998). The metal-poor clusters have on average higher metal abundance than the Milky Way globular clusters. The metal-rich clusters have slightly lower metallicities than previously thought, and are similar in abundance to the bulk of the halo stars in NGC 5128 (Harris \& Harris 2000), so it is tempting to speculate that these two populations are related to the same epoch of galaxy formation. In the present sample only 3 clusters have metallicities above solar, a fraction which is not inconsistent with the spread in $[\mathrm{Fe} / \mathrm{H}]$ caused by measurements errors. The fraction of very metal-rich clusters is lower than found by Harris et al. (1992). For the five metal-rich clusters in common between this work and Jablonka et al. (1996), the mean $[\mathrm{Fe} / \mathrm{H}]$ difference is 
only $0.06 \pm 0.16 \mathrm{dex}$ (standard deviation of the residuals), which represents an excellent agreement in view of the different instrument and methods.

We have also investigated whether there is a radial trend in the mean cluster metallicity, or in the relative number of metal-poor and metal-rich clusters. Although a radial gradient in the mean metallicity of the cluster system is not obvious, we do find some evidence for a gradual change in the mix of metal-poor and metal-rich clusters as a function of distance from the galaxy center (the percentage of metal-rich clusters being higher in the inner region).

\section{Conclusions}

We have presented new results from NTT multislit spectroscopy of 40 globular clusters in the inner region of NGC 5128, the nearest giant elliptical galaxy. Combined with previous results from multifibre spectroscopy of clusters in the halo of NGC 5128, these new data provide the first exhaustive spectroscopic information on the ages and metallicities of the globular clusters in this galaxy. Analysis of the Balmer and metal line indices shows that most of the clusters in NGC 5128 are similar in age to the Galactic globulars. We have found two clusters where strong hydrogen lines unambiguously indicate a young age. The metallicity distribution derived from spectroscopy is distinctly bimodal, thus confirming the presence of two cluster populations.

\section{References}

Bressan, A., Chiosi, C., \& Tantalo, R. 1996, A\&A, 311, 425

Frogel, J. A. 1984, ApJ, 278, 119

Harris, G. L. H., Geisler, D., Harris, H. C., \& Hesser, J. E. 1992, AJ, 104, 613

Harris, G. L. H., \& Harris, W. E. 2000, AJ, 120, 2423

Held, E. V., Federici, L., Testa, V., \& Cacciari, C. 1997, in ASP Conf. Ser. 116, The Nature of Elliptical Galaxies; 2nd Stromlo Symposium, ed. M. Arnaboldi, G. S. Da Costa \& P. Saha (San Francisco: ASP) , 500

Hesser, J. E., Harris, H. C., \& Harris, G. L. H. 1986, ApJ, 303, L51

Israel, F. P. 1998, A\&A Rev., 8, 237

Jablonka, P., Bica, E., Pelat, D., \& Alloin, D. 1996, A\&A, 307, 385

Kissler-Patig, M., Brodie, J. P., Schroder, L. L., Forbes, D. A., Grillmair, C. J., \& Huchra, J. P. 1998, AJ, 115, 105

Minniti, D., Alonso, M. V., Goudfrooij, P., Jablonka, P., \& Meylan, G. 1996, ApJ, 467, 221

Rejkuba, M. 2001, A\&A, 369, 812

Sharples, R. 1988, in IAU Symp. 126, The Harlow-Shapley Symposium on Globular Cluster Systems in Galaxies, ed. J. E. Grindlay \& A. G. Davis Philip (Dordrecht: Kluwer), 545

Soria, R., Mould, J. R., Watson, A. M., et al. 1996, ApJ, 465, 79

Worthey, G., Faber, S., González, J., \& Burstein, D. 1994, ApJS, 94, 687

Zepf, S. E., \& Ashman, K. M. 1993, MNRAS, 264, 611 
Zinn, R., \& West, M. J. 1984, ApJS, 55, 45

\section{Discussion}

P. Goudfrooij: As to the two candidate young clusters with strong Balmer lines, could you tell us where they are located in the galaxy?

E. Held: They are found at $\sim 2^{\prime}$ and $\sim 6^{\prime}$ from the galaxy center, respectively, projected quite far from the dust lane.

D. Forbes: What is the $[\mathrm{Mg} / \mathrm{Fe}]$ ratio of the clusters? What is their mean age ?

E. Held: As for the first question, we note that the relation between the $\mathrm{Mg}$ and Fe indices is very much consistent with the trend observed for Galactic globular clusters. However, subtle variations in the abundance ratio with respect to a solar composition cannot be stated with the resolution employed in this study. As for the mean age, it appears generally as old as for Galactic globular clusters, since the Balmer lines strengths are comparable. As it is generally the case for spectra of extragalactic clusters, the signal-to-noise does not allow a reliable discrimination of small age differences (e.g., from 10 to $13 \mathrm{Gyr}$ ).

J. Holtzman: To what extent do variations in horizontal branch morphology (i.e. the second parameter problem) affect age dating of clusters using the $\mathrm{H} \beta$ index?

E. Held: Models indicate that variations in the HB morphology do have some effect on the strengths of Balmer lines. However, the two candidate young clusters in NGC 5128 show Balmer line strengths much larger than accounted for by the subtle effects of HB morphology, which can only be explained by a young age. 